\title{
A Comparative Study of Early Initiation of Renal Replacement Therapy (RRT) VS Conservative Approach and/or Late Initiation of RRT in the Management of Severe Acute Kidney Injury (AKI)
}

\section{Kallol Bhattacharjee ${ }^{1 *}$, Akhil Agrawal ${ }^{2}$ and Sanjeeb Roy ${ }^{3}$ \\ ${ }^{1}$ Professor, Department of Medicine, Silchar Medical College and Hospital, India \\ ${ }^{2} \mathrm{PGT}$, Department of Medicine, Silchar Medical College and Hospital, India \\ ${ }^{3}$ PGT, Department of Medicine, Silchar Medical College and Hospital, India}

*Corresponding author: Kallol Bhattacharjee, Professor, Department of Medicine, Silchar Medical College and Hospital, Silchar, Assam, India, Tel: +918638787131; Email: kbsilchar64@ gmail.com

\section{Research Article \\ Volume 5 Issue 1}

Received Date: February 13, 2020

Published Date: February 21, 2020

DOI: $10.23880 /$ oajun-16000173

\section{Abstract}

Background: AKI is not a disease but rather a clinical syndrome with multiple etiologies as a result of multiple concurrent insults to the kidney and has been defined as a rapid decline in glomerular filtration rate that occurs over a period of minutes to days, with retention of blood urea nitrogen and serum creatinine ( $\mathrm{SCr}$ ).

Aims and Objectives: To evaluate the effect of early initiation of RRT versus conservative approach and/or late initiation of RRT on overall mortality and morbidity in cases of AKI.

Materials and Methods: The present study was a prospective single-centered, longitudinal, randomized trial conducted in the Department of Medicine at Silchar Medical College and Hospital, Silchar, Assam from 1st July 2016 to 30th June 2019. A total of 150 cases with AKI (KDIGO stage 2 and above) who underwent RRT were included in the study.

Results and Observations: Early initiation of RRT significantly reduced duration of renal support, median length of hospital stay. Metabolic abnormalities were more common in patients who received delayed RRT. Also a significantly higher number of patients recovered renal function at day 90 in early group as compared to delayed group.

Conclusion: In the management of critically ill patients diagnosed with AKI, initiation of early RRT is beneficial in terms of parameters like mortality, duration of hospital stay, and overall improvement in quality of life when compared with delayed initiation of RRT and/or conservative approach and it comes at a price which is negligible when weighed against the benefits.

Keywords: AKI; Critically Ill; Renal Function; KDIGO Stage; RRT

Abbreviations: SCr: Serum Creatinine; AKI: Acute Kidney Injury; ICU: Intensive Care Unit; RRT: Renal Replacement Therapy; SMCH: Silchar Medical College and Hospital; AKIKI: Artificial Kidney Initiation in Kidney Injury.

\section{Introduction}

Acute kidney injury (AKI) is not a disease but rather a clinical syndrome with multiple etiologies as a result of 


\section{Open Access Journal of Urology \& Nephrology}

multiple concurrentinsults to the kidneys. Acute kidney injury (AKI) is defined as a rapid downfall in glomerular filtration rate that occurs within minutes or may extend to days, with retention of blood urea nitrogen and serum creatinine ( $\mathrm{SCr}$ ). It is characterized by rise of serum creatinine by $0.3 \mathrm{mg} / \mathrm{dL}$ within 48 hours; or rise of serum creatinine by 1.5 times the baseline level, which is known or presumed to have occurred within the preceding 7 days; or a urine output of less than 0.5 $\mathrm{mL} / \mathrm{kg} / \mathrm{h}$ for 6 hours [1].

AKI is considered as one of the gravest and potentially fatal complications seen commonly in the critically ill patients admitted in an intensive care unit (ICU). Around 4 $\%$ of patients in the ICU develop AKI eventually and need renal replacement therapy (RRT) [2]. AKI is associated with a considerable amount of morbidity and up to $60 \%$ in-hospital mortality in its most severe form, necessitating an urgent renal replacement therapy (RRT) [3]. As there is no effective pharmacological treatment, the treatment of cases suffering from AKI is mainly supportive. The treatment modalities consist of managing hemodynamic and volume status, adjusting drug doses as per GFR, correction of electrolytes and acid-base imbalance and providing adequate nutrition.

In patients of severe AKI, RRT is life saving and absolutely necessary for managing volume overload, refractory hyperkalemia, acidosis and features of uremia while patiently waiting for the recovery of normal kidney function. Most physicians opine that RRT is necessary and not initiating RRT will be fatal in severely ill AKI patients. Conservative treatment has only been advocated as an option for less severe patients.

Rather than relying solely on laboratory data of hyperkalemia and raised creatinine or need to remove "retained fluid", the decision about timing of initiating RRT should bring into consideration the much broader clinical overview, wherein RRT could make a big difference in the final outcome including both patient and kidney survival. Initiating RRT early in AKI has the advantage of improving acid-base and electrolyte balance, avoidance of volume overload, prevention of more severe AKI complications and enhancement of toxin removal. However, an early RRT initiation results in exposure of the patient to hazards related to the dialysis procedure in terms of catheter placement and complications including volume depletion, hypotensive states, infection at fistula site, compromised kidney perfusion and other events related to ischemia. These events may prolong or worsen AKI, which may retard the chance of faster recovery of renal microanatomy and function [4]. However concrete data is sparse and conflictive regarding when to initiate RRT.

\section{Aims and Objectives}

To evaluate the effect of early RRT initiation versus conservative approach and/or delayed initiation of RRT on overall mortality and morbidity in cases of AKI.

\section{Materials and Methods}

Our study was a prospective single-centered, longitudinal, randomized trial undertaken in the Internal Medicine Department at Silchar Medical College and Hospital (SMCH), Silchar, Assam from $1^{\text {st }}$ July 2016 to $30^{\text {th }}$ June 2019. A total of 150 cases with AKI (KDIGO stage 2 and above) admitted at $\mathrm{SMCH}$ who underwent RRT were included in the study.

\section{Inclusion Criteria}

- Patient with confirmed diagnosis of AKI stage 2 and above (KDIGO criteria)

- Patient's age $>18$ years

\section{Exclusion Criteria}

- Patient's age $<18$ years

- Patients with pre-existing renal disease viz preexisting chronic kidney diseases like glomerulonephritis, vasculitis, interstitial nephritis, postrenal obstruction, or those with hemolytic uremic syndrome, thrombotic thrombocytopenic purpura were excluded. Pregnancy, prior kidney transplantation and hepatorenal syndrome were also excluded from the study.

- Patients denying consent.

\section{Randomization and Intervention}

AKI was diagnosed on the basis of KDIGO criterion. Creatinine measurements were taken twice per day. Every patient was put on a urinary catheter and urine output was recorded every 6 hourly. Prior to randomization, written and informed consent were taken from all participants included in the study. Cases satisfying the parameters for inclusion and exclusion criteria were randomized in either of the two treatment groups. Both treatment groups consisted of 75 patients each. Early RRT was initiated when patient had KIDGO stage 2 AKI (serum creatinine level 2.0-2.9 times baseline and/or urine output $<0.5 \mathrm{ml} / \mathrm{kg} / \mathrm{h}$ for 12 hours) and Late RRT was initiated when patient had KIDGO stage 3 AKI (serum creatinine 3.0 times baseline OR Increase in serum creatinine to $>4.0 \mathrm{mg} / \mathrm{dl}$ and/or urine output $<0.5$ $\mathrm{ml} / \mathrm{kg} / \mathrm{h}$ for 24 hours or anuria for 12 hours) or any of the classical indications of initiation of RRT.

Patients were subjected to intermittent hemodialysis in both groups. Renal-replacement therapy was discontinued if the spontaneous urine output exceeded $1000 \mathrm{ml}$ in 24 hours 


\section{Open Access Journal of Urology \& Nephrology}

without any diuretics or if the urine output exceeded $2000 \mathrm{ml}$ in 24 hours in cases on diuretic therapy. Discontinuation of RRT was mandatory when urine output was enough to allow for a spontaneous decline in the serum creatinine levels.

Statistical analyses were done using SPSS software version 20.0 and a $p$-value $<0.05$ was considered to be statistically significant.

\section{Outcomes}

The primary end-point was mortality in 90 days and organ dysfunction. The secondary outcomes measures were duration of RRT, improvement of kidney function, length of hospital stay including the period of stay in ICU (Intensive Care Unit) and dialysis dependency.

\section{Results}

A total of 229 patients met criterion for the trial, of which 150 were randomized into groups of 75 each for early RRT and delayed RRT. The demographic characteristics of patients were well matched for both the groups. At the onset of RRT initiation, the delayed group had higher serum creatinine and urea levels with significantly lower urine output in comparison with the early group (Table 1).

\begin{tabular}{|c|c|c|c|}
\hline & & EARLY (n=75) & DELAYED (n=75) \\
\hline Age & & $59.2 \mathrm{yrs}$ & $57.9 \mathrm{yrs}$ \\
\hline \multirow{2}{*}{ Sex } & Male & $46(61.3 \%)$ & $44(58.7 \%)$ \\
\cline { 2 - 4 } & Female & $29(38.7 \%)$ & $31(41.3 \%)$ \\
\hline Baseline Creatinine (mean) & & $2.1 \mathrm{mg} / \mathrm{dl}$ & $2.9 \mathrm{mg} / \mathrm{dl}$ \\
\hline Serum Potassium & & $4.9 \mathrm{mmol} / \mathrm{l}$ & $5.0 \mathrm{mmol} / \mathrm{l}$ \\
\hline Serum Bicarbonates & & $19.3 \mathrm{mmol} / \mathrm{l}$ & $19.1 \mathrm{mmol} / \mathrm{l}$ \\
\hline Estimated GFR (mean) & & $33.6 \mathrm{ml} / \mathrm{min}$ & $36.4 \mathrm{ml} / \mathrm{min}$ \\
\hline \multirow{3}{*}{ Comorbidities } & Hypertension & $56(74.6 \%)$ & $59(78.6 \%)$ \\
\cline { 2 - 4 } & Diabetes & $39(52 \%)$ & $37(49.3 \%)$ \\
\cline { 2 - 4 } & Congestive Cardiac Failure & $17(22.7 \%)$ & $18(24 \%)$ \\
\cline { 2 - 4 } & Chronic Obstructive Pulmonary Disease & $33(44 \%)$ & $36(48 \%)$ \\
\cline { 2 - 4 } Treatment received & Mechanical Ventilation & $40(53.3 \%)$ & $42(56 \%)$ \\
\cline { 2 - 4 } & Vasopressor drugs & $49(65.3 \%)$ & $51(68 \%)$ \\
\hline
\end{tabular}

Table 1: Baseline characteristics of patients who underwent RRT.

The follow-up data at Day 90 revealed that there were total of 69 deaths in the study population. Early initiation of RRT significantly brought down the 90-day mortality (Figure 1) in comparison with late initiation of RRT (34 of 75 patients [45.3\%] in the early group versus 41 of 75 patients [54.6\%] in the delayed group) $\mathrm{p}=.03$ (i.e.p $<0.05$ ).
Early RRT initiation significantly reduced the period of time of renal support, median duration of hospital stay and ICU stay. Metabolic abnormalities were commoner in patients who received delayed RRT. Also a significantly higher number of patients recovered renal function at day 90 in early group as compared to delayed group.

\begin{tabular}{|c|c|c|c|c|}
\hline & & Early group & Delayed group & p-value \\
\hline Mortality at Day 90 & & $34(45.3 \%)$ & $41(54.6)$ & 0.03 \\
\hline \multirow{2}{*}{ Median duration of ICU stay } & Survivors & 11 days & 12 days & \multirow{2}{*}{0.09} \\
\cline { 2 - 4 } & Non-survivors & 6 days & 6 days & \multirow{2}{*}{0.04} \\
\hline \multirow{2}{*}{ Median duration of hospital stay } & Survivors & 16 days & 19 days & 6 days \\
\cline { 2 - 4 } & Non-survivors & 6 days & \multicolumn{2}{|c}{} \\
\hline
\end{tabular}




\section{Open Access Journal of Urology \& Nephrology}

\begin{tabular}{|c|c|c|c|c|}
\hline \multirow{4}{*}{ Complications related to AKI or RRT } & Anaemia & $29(38.3 \%)$ & $31(41.3 \%)$ & 0.06 \\
\cline { 2 - 5 } & Thrombocytopenia & $21(28 \%)$ & $22(29.3 \%)$ & 0.08 \\
\cline { 2 - 5 } & Haemorrhage & $12(16 \%)$ & $12(16 \%)$ & \\
\cline { 2 - 5 } & Hyperkalemia & $36(48 \%)$ & $46(61.33 \%)$ & 0.04 \\
\cline { 2 - 5 } & Hypokalemia & $10(13.3 \%)$ & $9(12 \%)$ & 0.06 \\
\cline { 2 - 5 } & Hyperphosphatemia & $26(34.6 \%)$ & $28(37.3 \%)$ & 0.07 \\
\hline Recovery of Renal function at Day 90 & & $40(90.9 \%)$ & $29(82.85 \%)$ & 0.01 \\
\hline
\end{tabular}

Table 2: Primary and Secondary Outcome.

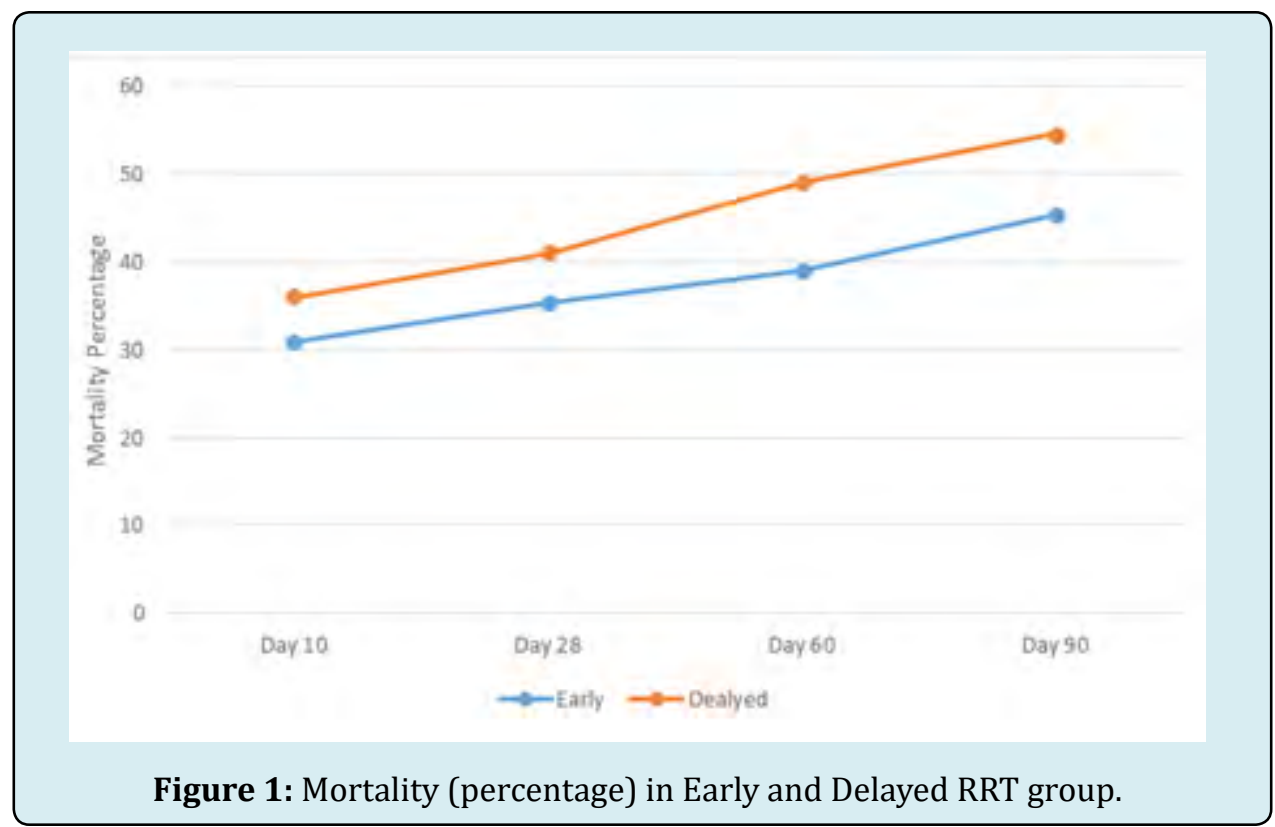

\section{Discussion}

The early RRT group (45.3\%) had significantly less mortality compared to the delayed RRT group (54.6\%) and a greater number of patients who survived AKI had recovered kidney function at day 90 . The hospital stay was smaller in patients with early RRT. The findings can be explained by the fact that early RRT leads to better fluid overload management and better metabolic and uremic control. A single-centred trial was performed by Jamale, et al. [5] in western India wherein earlier starting of dialysis was initiated only when serum creatinine levels were raised to $7 \mathrm{mg} / \mathrm{dl}$ whereas the usual-start dialysis patients received dialysis when clinically judged by treating nephrologist taking into consideration other indications. The study data did not find support in earlier initiation of RRT in community acquired AKI. The study found less mortality than predicted in study population $(20.5,12.2 \%)$ in the two groups respectively.

In ELAIN (Early Versus Late Initiation of RRT in Critically Ill Patients with AKI) Trial enrolling 231 critically ill patients with KDIGO stage 2 AKI, duration of RRT, mechanical ventilation, period of hospital stay and mortality at day 90 was significantly lower among the cases randomized to the early initiation group in comparison with those in the delayed initiation group [6]. The mortality at day $90(39.3 \%, 54.7 \%)$ was similar to that found in our study $(41.3 \%, 50.7 \%)$. A randomized clinical trial by Suhagara S, et al. in 2014 observed that RRT if initiated early resulted in a decreased mortality on head to head comparison with cases where RRT was initiated late. The study was related to evaluation of the effect of early RRT initiation in patients numbering 28 who developed AKI after a cardiac surgery. They proposed that the timing for start of RRT for management of AKI following cardiac surgery should be determined by the levels of decline in urinary output and not by increase in serum creative levels [7]. The systematic review and meta-analysis by Karvellas CJ, et al. [8] concluded that earlier initiation of RRT in the critically ill patients resulted in a positive effect on survival. The conclusion was however based on heterogenous studies comprising of variable quality and based on two randomized trials $[7,9]$. Utilising data from the Beginning and Ending 


\section{Open Access Journal of Urology \& Nephrology}

Supportive Therapy for the Kidney (BEST Kidney) study, which was an observational study including critically ill patients with AKI in 54 ICUs spread across 23 countries, Bagshaw, et al. [10] compared the outcomes of cases who were initiated on dialysis 'early' or 'late' using several measures of timing. No differences in crude or adjusted mortality were observed between the early and late groups. This study enlightens some of the hurdles with observational studies regarding timing of RRT initiation. Inference derived from such studies result in situations which are dependent on the definitions used for 'early' and 'late', which vary considerably across available literature [10].

The Artificial Kidney Initiation in Kidney Injury (AKIKI) Trial, which was an unblinded French multicenter, randomized trial, enrolled 620 critically ill patients who had KDIGO stage $3 \mathrm{AKI}$. The trial observed no significant difference of outcome regarding mortality when compared with early and a delayed initiation of RRT. A delayed strategy negated the requirement of RRT in a considerable number of cases. However the trial did not recommend 'wait and see' approach to be a safer option for the treatment of AKI concluding careful surveillance to be mandatory when deciding to delay RRT in cases with severe AKI so as to detect any complication and if required renal-replacement therapy is to be initiated without delay [11].

In another randomized clinical trial, Bouman, et al. [12] had studied 106 cases with AKI and randomized them to early or late initiation of RRT. Patients in the early group were subjected to RRT soon after satisfying criteria for AKI, whereas delayed RRT initiation was undertaken when patients were complicated with hyperkalemia or pulmonary edema or plasma urea levels higher than $440 \mathrm{mmol} / \mathrm{L}$. No significant difference was observed in mortality [12]. Similarly no significant difference in 90 day survival between early and late initiation of RRT was observed in a study conducted by Wald and colleagues in 2015. Of the total patients receiving RRT, $25 \%$ of them could have survived without RRT and this mode of therapy instituted as per the judgement of treating physician could not alter the course of the disease [13]. A cohort study of 1213 patients treated with continuous RRT by Dr Christiansen et al do not opine the superiority of early RRT initiation to late RRT initiation. Although early RRT initiation resulted in a higher short-term mortality, there was no correlation between timing and longterm outcome [14].

The difference in results could be attributed to variation in defining criteria for 'early RRT' and also heterogenous study designs of various trials. The trials also differ in dosage and type of hemodialysis. The beneficial effects of early RRT initiation are because of rapid metabolic or uremic control and highly effective prevention and management of volume overload [15]. Early RRT initiation prior to the occurrence of severe AKI may minimize kidney-specific and non-kidney organ damage from uremia, fluid overload, acidemia and systemic inflammatory response and could potentially result into rapid recovery of kidney function and improved survival $[16,17]$. The counterargument against early RRT is "more intensive therapy comes at an incremental cost without a significant rise in the quality of life" [18]. At the same time, a strategy of early RRT might subject patients who would recover renal function with conservative treatment alone to the potential risks and complications associated with RRT.

However, AKI substantially increases the risk of death in cases never receiving RRT. Although there might be a risk of "unnecessary and avoidable" RRT, the risk associated with not providing RRT is even bigger and that is the bottom line.

\section{Limitations of the Study}

The study was a single centred study covering a limited geographical area. A multi centric approach with the coverage of wider geographical location covering diverse sections of the society would have been appropriate for this study. Further multi-centric trials with increasing number of patients using this intervention are warranted to evolve clear guidelines.

\section{Conclusion}

In managing critically ill patients diagnosed to have AKI, initiation of early RRT is beneficial in terms of parameters such as mortality, duration of hospital stay, and overall improvement in quality of life in comparison with delayed RRT initiation and/or conservative approach and it comes at a price which is minimum when weighed against the benefits.

\section{References}

1. Kidney International Supplements (2012) 2: 2.

2. Uchino S, Kellum JA, Bellomo R, Doig GS, Morimatsu H, et al. (2005) Acute renal failure in critically ill patients: a multinational, multicar study. JAMA 294(7): 813-818.

3. Jun M, Heerspink HJ, Ninomiya T, Gallagher M, Bellomo $R$, et al. (2010) Intensities of renal replacement therapy in acute kidney injury: a systematic review and metaanalysis. Clin J Am Soc Nephrol 5(6): 956-963.

4. Bielopolski D, Zadeh KK (2016) Conservatism strikes back: later is better than earlier dialysis for acute kidney injury. J Thorac Dis 8(9): 2415-2419.

5. Jamale TE, Hase NK, Kulkarni M, Pradeep KJ, Keskar V, et al. (2013) Earlier-start vs usual-start dialysis in patients with community-acquired acute kidney injury 


\section{Open Access Journal of Urology \& Nephrology}

a randomized controlled trial. Am J Kidney Dis 62(6): 1116-1121.

6. Zarbock A, Kellum JA, Schmidt C, Aken HV, Wempe C, et al. (2016) Effect of Early vs Delayed Initiation of Renal Replacement Therapy on Mortality in Critically Ill Patients With Acute Kidney Injury: The ELAIN Randomized Clinical Trial. JAMA 315(20): 2190-2199.

7. Sugahara S, Suzuki H (2004) Early start on continuous hemodialysis therapy improves survival rate in patients with acute renal failure following coronary bypass surgery. Hemodial Int 8(4): 320-325.

8. Karvellas CJ, Farhat MR, Sajjad I, Mogensen SS, Leung AA, et al. (2011) A comparison of early vs late initiation of renal replacement therapy in critically ill patients with acute kidney injury: a systematic review and metaanalysis. Crit Care 15(1): 72.

9. Korkeila M, Ruokonen E, Takala J (2000) Costs of care, long-term prognosis and quality of life in patients requiring renal replacement therapy during intensive care. Intensive Care Med 26(12): 1824-1831.

10. Bagshaw SM, Uchino S, Bellomo R, Morimatsu H, Morgera $S$, et al. (2009) Timing of renal replacement therapy and clinical outcomes in critically ill patients with severe acute kidney injury. J Crit Care 24(1): 129-140.

11. Liu KD, Palevsky PM (2016) RRT in AKI: Start Early or Wait?. Clin J Am Soc Nephrol 11(10): 1867-1871.

12. Bouman CS, Oudemans-Van Straaten HM, Tijssen JG, Zandstra DF, Kesecioglu J (2002) Effects of early high- volume continuous venovenous hemofiltration on survival and recovery of renal function in intensive care patients with acute renal failure: a prospective, randomized trial. Crit Care Med 30(10): 2205-2211.

13. Wald R, Adhikari NK, Smith OM, Weir MA, Pope K, et al. (2015) Comparison of standard and accelerated initiation of renal replacement therapy in acute kidney injury. Kidney Int 88(4): 897-904.

14. Christiansen S, Christensen S, Pedersen L, Gammelager H, Layton JB, et al. (2017) Timing of renal replacement therapy and long-term risk of chronic kidney disease and death in intensive care patients with acute kidney injury. Crit Care 21: 326.

15. Gibney N, Hoste E, Burdmann EA, Bunchman T, Kher V, et al. (2008) Timing of initiation and discontinuation of renal replacement therapy in AKI: unanswered key questions. Clin J Am Soc Nephrol 3(3): 876-880.

16. Clark WR, Letteri JJ, Uchino S, Bellomo R, Ronco C (2006) Recent clinical advances in the management of critically ill patients with acute renal failure. Blood Purif 24(5-6): 487-498.

17. Matson J, Zydney A, Honoré PM (2004) Blood filtration: new opportunities and the implications of systems biology. Crit Care Resusc 6(3): 209-217.

18. De Smedt DM, Elseviers MM, Lins RL, Annemans L (2012) Economic evaluation of different treatment modalities in acute kidney injury. Nephrol Dial Transplant 27(11): 4095-4101. 